\title{
Endoscopic ultrasound-guided biopsy in chronic liver disease: a randomized comparison of 19-G FNA and 22-G FNB needles $\square$
}

\section{(ㄷ)(우우}

\author{
Authors \\ Shaffer R. S. Mok, David L. Diehl, Amitpal S. Johal, Harshit S. Khara, Bradley D. Confer, Prashant R. Mudireddy, Alicia \\ H. Kovach, Mia M. Diehl, H. Lester Kirchner, Zong-Ming E. Chen
}

Institution

Geisinger Medical Center, Department of Gastroenterology and Hepatology, Division of Interventional Endoscopy, Danville, Pennsylvania, United States

submitted 23.3.2018

accepted after revision 11.6 .2018

\author{
Bibliography \\ DOI https://doi.org/10.1055/a-0655-7462 | \\ Endoscopy International Open 2019; 07: E62-E71 \\ (c) Georg Thieme Verlag KG Stuttgart · New York \\ ISSN 2364-3722
}

\section{Corresponding author}

Shaffer R. S. Mok, MD, MBS, Geisinger Medical Center, Department of Gastroenterology and Hepatology, Division of Interventional Endoscopy, 100 North Academy Ave,

Danville, PA, 17822

Fax: +1-570-271-6852

mok.shaffer@gmail.com

\section{ABSTRACT}

Background and study aims Endoscopic ultrasoundguided liver biopsy uses a 19-gauge (G) needle for parenchymal liver biopsies. We evaluated tissue yields with a $22 \mathrm{G}$ fine-needle biopsy (FNB) versus 19G FNA fine-needle aspirate (FNA) device.

Patients and methods Biopsies were obtained from 20 patients using the $19 \mathrm{G}$ FNA and 22G FNB randomizing each in a cross-over fashion with a blinded outcome assessor. Tissue adequacy for histologic evaluation was the primary outcome, or the proportion of specimens obtaining pathologic diagnosis (portal structures $\geq 5$ or length of the longest piece $\geq 15 \mathrm{~mm}$ ). Additional secondary outcomes included portal and centrilobular inflammation/fibrosis, length of the longest piece, aggregate specimen length, and small $(<5 \mathrm{~mm})$, medium $(5-8 \mathrm{~mm})$ and large $(>8 \mathrm{~mm})$ fragments. Results were compared in a per needle basis. Patients with cirrhosis were excluded.

Results Eighty biopsies (40 each 19G FNA and 22G FNB) were obtained. Tissue adequacy was greater for the 19G FNA (88\%) versus 22G FNB (68\%), $(P=0.03)$. There was no difference in total portal structures for the 19G FNA (7.4) and 22G FNB (6.1), $(P=0.28)$.

There was no difference in pre-processing outcomes. After processing, length of the longest piece was higher for the 19G FNA $(9.1 \mathrm{~mm})$ versus 22G FNB $(6.6 \mathrm{~mm}),(P=0.02)$. More total post-processing small fragments 29.9 versus 20.7, $(P=0.01)$ and fewer large fragments 1.0 versus 0.4 for the 22G FNB $(P=0.01)$ were detected.

Conclusions Tissue adequacy was higher for the 19G FNA versus $22 \mathrm{G}$ FNB needle. The 22G FNB needle produced samples more prone to fragmentation during specimen processing.

\section{Clinical.Trials.gov}

NCT02967991

TRIAL REGISTRATION: Randomized, single-center, prospective cross-over study with a single-blinded outcome assessor NCT02967991 at clinicaltrials.gov

\section{Introduction}

Serologic markers and diagnostic imaging, such as fibroscan, are often utilized to evaluate chronic liver diseases [1-4]. Due to advancements in diagnostic imaging modalities and serologic testing, the demand for procuring hepatic tissue has declined. Despite the benefit of such diagnostic imaging, it is still common for the clinician to require additional means for deter- mining the etiology or stage of a patient's chronic liver disease, thus liver biopsy remains an important procedure.

Percutaneous and transjugular liver biopsy, the two widely used access routes for obtaining liver histology, utilize percussion or imaging to localize a suitable biopsy site [5-7]. Endoscopic ultrasound-guided liver biopsy (EUS-LB) is another method of acquiring liver tissue $[8,9]$. The feasibility of EUS-LB for liver lesions has been validated in several studies, yielding 


\begin{tabular}{ll}
\hline ABBREVIATIONS \\
EUS-LB & EUS-Guided Liver Biopsy \\
g & gauge \\
FNA & Fine Needle Aspiration \\
FNB & Fine Needle Biopsy \\
LLP & Length of the Longest Piece \\
CLD & Chronic Liver Disease \\
EUS & Endoscopic Ultrasound \\
EUS-FNA & Endoscopic Ultrasound-Fine Needle Aspiration \\
LB & Liver Biopsy \\
IRB & Investigational Review Board \\
DSMB & Data and Safety Monitoring Board \\
INR & International Normalized Ratio \\
mL & milliliters \\
mm & millimeters \\
IHC & Immunohistochemical \\
ASL & Aggregate Specimen Length \\
CI & Confidence Interval \\
BMI & Body Mass Index \\
NASH & Non-alcoholic Steatohepatitis \\
PBC & Primary Biliary Cholangitis \\
AE & Adverse Events \\
ED & Emergency Department \\
IR & Interventional Radiology \\
H \& E & Hematoxylin and Eosin \\
Pap & Papanicolau \\
RPMI & Roswell Park Memorial Institute \\
ROSE & Rapid On-Site Cytologic Evaluation \\
RBC & Red Blood Cell \\
SD & Standard Deviation \\
CI & Confidence Interval \\
PAS & Periodic Acid Schiff \\
& \\
\hline
\end{tabular}

excellent diagnostic results [8-12]. A comparison of percutaneous, transjugular, and EUS-LB demonstrated similar, and in some instances improved, sample yields for EUS-LB [13-15]. In addition, EUS-LB has a theoretical advantage of allowing for improved patient comfort, less post-procedural pain and the ability to evaluate the upper gastrointestinal tract and pancreaticobiliary anatomy, should abdominal pain be present or varices suspected. A 22G FNB EUS core needle is also available for use but there are no data available comparing these needles. In this study, we compared use of a 22G fine-needle biopsy (FNB) needle to the standard 19G FNA fine-needle aspiration (FNA) needle for EUS-LB. We hypothesized that the 22G FNB needle would demonstrate noninferior tissue adequacy when compared with the 19G FNA needle.

\section{Patients and methods}

\section{Study design}

This is a randomized cross-over study with a blinded outcome assessor of patients undergoing liver biopsy at a tertiary care center in the United States. This study was approved by our In- stitutional Review Board (IRB 2016-0281). This trial was registered online at Clinicaltrials.gov (NCT02967991) prior to enrollment of any participants. A data and safety monitoring board composed of non-participating members of our division provided oversight during this trial.

\section{Patients}

Patients eligible for study participation included individuals undergoing EUS-LB for diagnosis or staging of chronic liver diseases. In particular, we included individuals undergoing LB for elevated liver enzymes of unknown origin, those with existing non-alcoholic steatohepatitis (NASH), alcohol-induced hepatitis, hepatitis $C$, metabolic liver disease or autoimmune chronic liver disease, requiring staging. Patients were excluded if they were under 18 years of age, pregnant, unable to sign informed consent, had a platelet count under $50,000 / \mu \mathrm{L}$, international normalized ratio (INR) $>1.5$, or diagnosis of cirrhosis. We excluded individuals with cirrhosis because of their risk of coagulopathy and potential bleeding, as well as the likelihood of fragmentation of specimens with the EUS method. Although liver biopsy is performed by other means at our institution, techniques other than EUS-LB were excluded. Patients also were excluded if they were using antiplatelet agents (excluding aspirin) or anticoagulants and could not stop these medications for the procedure. All patients underwent laboratory testing (complete blood count and INR) prior to their EUS-LB.

\section{Study procedures}

Informed consent for the EUS-LB procedure as well as for participation in this study was obtained from all participants. No antibiotics were given prior to the procedure. Biopsies of both liver lobes, using both a $19 \mathrm{G}$ EUS-FNA needle (Expect Flexible 19G, Boston Scientific, Marlborough, Massachusetts, United States) and a 22G FNB needle (SharkCore, Covidien-Medtronic Inc, Minneapolis, Minnesota, United States) were performed in all participants.

The initial needle puncture size (19G FNA or 22G) was randomized in a 1:1 fashion. All subjects underwent biopsies with each needle in the left and right lobes and were randomized to the needle used for first puncture. They were then crossed over to the other needle and the biopsy repeated. This randomization schedule was created by a computerized random number generator and was kept on password-protected computers in each endoscopy room only accessible to a study member, who was not participating in the procedure itself. This computergenerated randomization would be pulled up after subject enrollment, by a member of the study team not involved in the procedure, thus manintaining allocation concealment. EUS-LBs were performed by one of three experienced therapeutic endoscopists (DLD, ASJ and HSK) who had each performed at least 100 EUS-LBs. Subjects were then crossed over to the alternate needle for a second pass. The initial pass was randomized.

The endosonographic study was conducted with a linear array echoendoscope (GF-UCT140-AL5 or GF-UCT180; Olympus America, Center Valley, Pennsylvania, United States). The left lobe was described as liver parenchyma identified a few centi- 
meters below the gastroesophageal junction with the echoendoscope torqued counterclockwise within the stomach. The right lobe was considered the large area of liver tissue seen through the duodenal bulb after torqueing counterclockwise, near the gallbladder [16].

The needle was prepared prior to biopsy by removing the stylet, flushing heparin through the needle lumen, and setting the suction device on full $(20 \mathrm{~mL})$ and attaching it to the needle hub. The primed and prepared needle was then inserted into the echoendoscope and color doppler imaging used to ensure lack of vascular or biliary structures in the trajectory of the needle. A transgastric approach was used to obtain samples from the left lobe of the liver and a transduodenal approach was used for the right lobe. Once adequate liver parenchymal penetration was achieved with the needle $(\sim 2-6 \mathrm{~cm})$, full suction was applied with the $20-\mathrm{mL}$ vacuum syringe. One pass consisted of a total of 7 to 10 to-and-fro needle motions in various planes, with the fanning technique applied under direct and continuous endosonographic visualization of the tip of the needle ( $\triangleright$ Video 1$)$.

The needle was then removed from the echoendoscope and the specimen was pushed from the needle with the stylet directly into a microsieve (CellSafe Biopsy Capsule, Cancer Diagnostics, Durham, North Carolina, United States) and blood washed away from the specimen with a gentle saline rinse. Due to the prior heparin priming, the majority of the blood does not clot and could be washed through, leaving nearly pure liver cores. The endosonographer looked for multiple fragments of light brown tissue approximately 5 to $15 \mathrm{~mm}$ in length, making rapid on-site pathologic assessment unnecessary as core tissue was obtained. The tissue cores were then "floated" off the microsieve into formalin solution. Then, a second pass was made from the same region of the liver using the alternate needle. The biopsy process was then repeated in the right liver lobe following the same process as above, with the first pass randomized as well. All patients were closely observed in the recovery area for 1 hour after the procedure.

\section{Sample processing}

Tissue samples were left in formalin for at least 1 hour before processing. The contents of the formalin jar were poured into a Petri dish, and visible cores of liver tissue were identified by the blinded surgical pathology technician. These fragments were arranged in a linear fashion on lens paper and photographed alongside a ruler to determine pre-processing tissue lengths. Samples from each patient were submitted separately. The tissue was processed in standard fashion, and slide blanks were made (5- $\mu \mathrm{m}$ tissue thickness). These blanks were stained with hematoxylin and eosin, trichrome, and reticulin, with other special stains done as needed. The slides were digitized using a whole slide scanner (ScanScope CS; Aperio Technologies, Inc, Vista, California, United States), the digitized images were used for quantitative analysis (eSlide Manager; Aperio Technologies, Inc). The digitized images contained no patientidentifying information or any information to relate which of the specimens came from the same patient.

\section{Outcomes}

The primary outcome was tissue adequacy for histologic evaluation, defined as the proportion of cases for which $\geq 5$ portal structures or a length $\geq 15 \mathrm{~mm}$ was obtained by EUS-LB tissue, allowing a clinical diagnosis [17]. Two fellowship-trained gastrointestinal pathologists (ZMEC and a second interpreter) evaluated tissue adequacy from the blinded biopsy slides. These pathologists used a specific scoring system evaluating the below parameters, which was used for all samples ( $\triangleright$ Appendix $\mathbf{1}$ ). For the pathologist's determination of adequacy, 19G FNA and 22G FNB specimens were placed into a random order for blinded interpretation. Should a discrepancy exist between our pathologists, the slides would be reviewed by each pathologist together and discussed for consensus. Also during their blinded readings, our pathologists evaluated secondary outcomes including portal inflammation, portal and centrilobular fibrosis. For these outcomes, they rated these parameters and determined whether these histologic aspects were: 1) definitely present; 2) questionably present; or 3) not present.

Additional secondary outcomes included total number of portal structures (partial or complete), which was defined by presence of hepatic artery with smooth muscle cells, portal vein with epithelial lining and bile duct with cuboidal cell lining. Visible blood, defined by presence of red blood cells within the histology specimen, and visible core tissue, defined as present tan pieces of tissue after procuring/washing the sample as seen on our microsieve, were both other secondary outcomes. Pre- and post-processing tissue outcomes included the length of the longest piece in millimeters, which was defined by the single tissue fragment with the longest measured length. Preand post-processing aggregate specimen length was also measured, defined as the total measured length of all tissue fragments. Finally, we evaluated the degree of fragmentation for which we divided obtained tissue into small $(<5 \mathrm{~mm})$, medium $(5-8 \mathrm{~mm})$ and large tissue fragments $(>8 \mathrm{~mm})$.

Pre-processing photographs of biopsy fragments were measured using Image] software (https://imagej.nih.gov/ij/). Post-processing measurements were obtained using the "ruler" function of the eslide Manager software. Quantification of sample length $(\mathrm{mm})$ and portal structures was performed by three of the investigators (SM, MD and DD), annotating the digital images with the software. Aggregate specimen length was calculated by measuring each fragment on this software platform and adding the results together.

Adverse events (AE) were tracked using telephone call back $[18,19]$. Patients were followed-up with by a phone call at 1 day and 1 week after the procedure.

\section{Statistical analysis}

This is a pilot study with a goal of obtaining estimates to design a larger study. The precision, as measured by the half-width of a $95 \%$ confidence interval $(\mathrm{Cl})$ was used to select the sample size. It was assumed tissue adequacy for the control group was $88 \%$. With a sample size of 40 subjects, the study is able to estimate a $95 \% \mathrm{Cl}$ with a half-width of $10 \%$ (i. e., $78 \%-98 \%$ ). 


\section{Assessed for eligibility $(n=84)$}

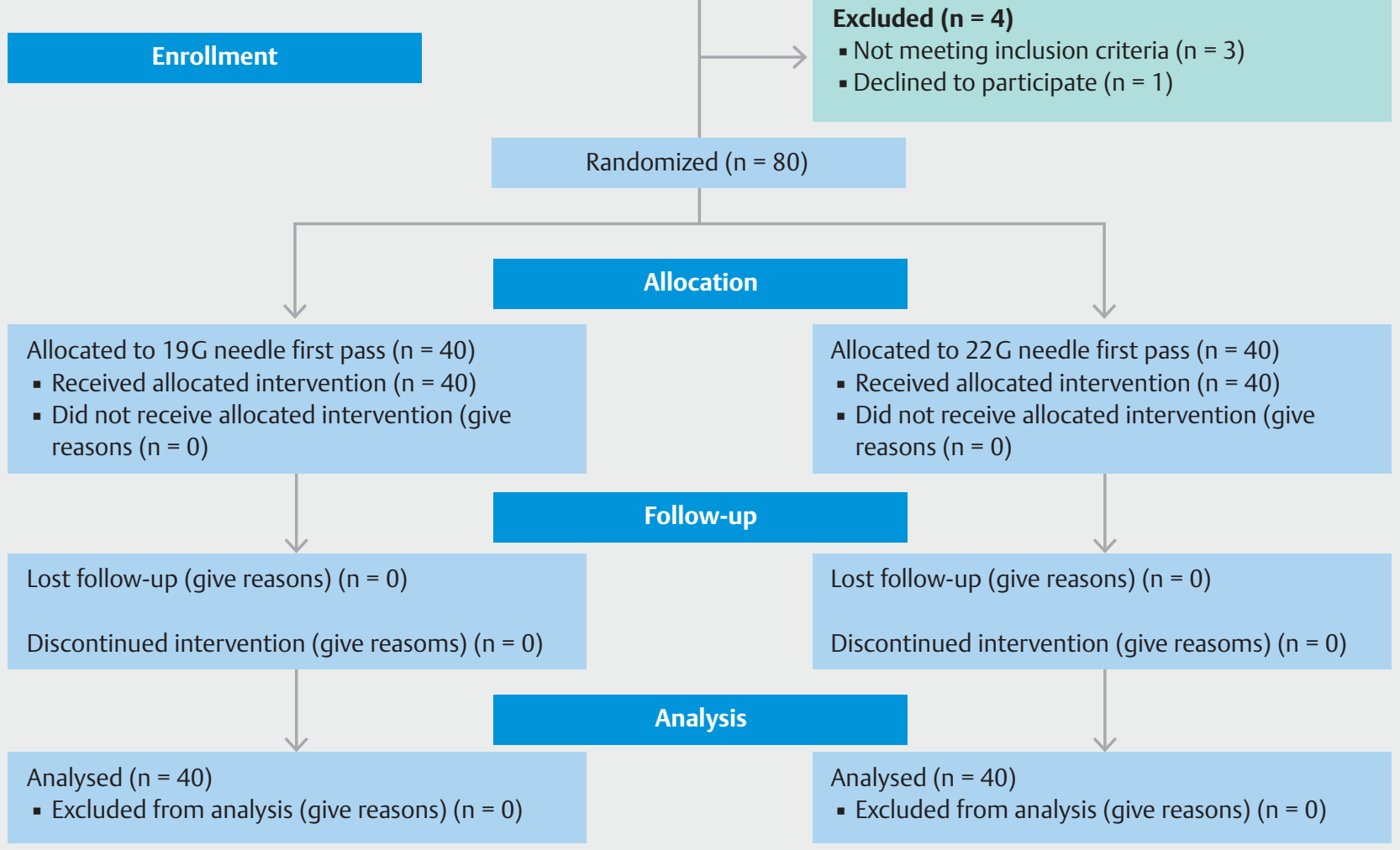

- Fig. 1 Flow diagram with enrollment and outcomes.

Descriptive statistics were summarized for all demographic data including age, gender, and reason for EUS-LB. Continuous variables are reported as median and interquartile range, and categorical variables are shown as frequency and percentage. The $95 \% \mathrm{Cl}$ was estimated for the primary outcome of tissue adequacy. Even though this is a pilot study, statistical inference was performed to compare the wet and dry heparin groups to the control group. This was accomplished using McNemar's Chi square test for binary variables and the Wilcoxon Signed Rank test for continuous variables. Data analysis was performed using SAS v9.4 (SAS Institute Inc. Cary, NC). $P<0.05$ was considered statistically significant.

\section{Results}

From August 2016 through November 2016, 80 biopsies were performed in consecutive patients. Fifty percent of patients underwent first biopsy with a 19G FNA needle and $50 \%$ underwent first biopsy with the 22G FNB needle ( $\triangleright$ Fig. 1). Demographic data are summarized in $>$ Table 1 . All patients completed both 1-day and 1-week follow-up. Of note, the average BMI of the sample was 31.2.

All data were reported as a per needle basis. The most common diagnosis was NASH in $50 \%$, followed by primary biliary cholangitis (PBC) in $20 \%$ ( $\triangleright$ Table 1 ).
- Table 1 Demographics.

\begin{tabular}{|l|l|l|}
\hline $\mathbf{n}=\mathbf{4 0}$ & & Mean (SD) or $\mathbf{n}(\%)$ \\
\hline Mean age & & $50.5(18.4)$ \\
\hline Sex & Female & $30(75 \%)$ \\
\hline & Male & $10(25 \%)$ \\
\hline \multirow{2}{*}{ Race } & White & $40(100 \%)$ \\
\hline \multirow{2}{*}{ Indications } & BMI (kg/m²) & $31.2(7.1)$ \\
\hline \multirow{2}{*}{} & Elevated LFT unknown etiology & $28(70 \%)$ \\
\hline & Hepatitis C Staging & $4(10 \%)$ \\
\hline & NASH staging & $2(5 \%)$ \\
\hline & Alcohol Hepatitis & $2(5 \%)$ \\
\hline & PBC post-treatment & $2(5 \%)$ \\
\hline & Abnormal ceruloplasmin level & $2(5 \%)$ \\
\hline
\end{tabular}

m, meters; kg, kilograms; BMI, body mass index; LFT, liver function test; PBC, primary biliary cholangitis 
- Table 2 Comparision of specimen parameters for the $19 \mathrm{G}$ versus $22 \mathrm{G}$ needles.

\begin{tabular}{|c|c|c|c|c|}
\hline \multirow[t]{2}{*}{$n=80$} & & 19G FNA & $22 \mathrm{G} F N B$ & \\
\hline & & mean (SD) or $\mathbf{n}(\%)$ & mean (SD) or $\mathbf{n}(\%)$ & $P$ value \\
\hline \multicolumn{2}{|l|}{ Adequate specimen ${ }^{1}$} & $35(88 \%)$ & $27(68 \%)$ & 0.03 \\
\hline \multicolumn{2}{|l|}{ Total with portal tracts $\geq 5$} & 32 & 23 & 0.03 \\
\hline \multicolumn{2}{|c|}{ Total with length of the longest piece $\geq 15 \mathrm{~mm}$} & 15 & 11 & 0.34 \\
\hline \multirow[t]{7}{*}{ Pre-processing ${ }^{2}$} & Total visible core & $40(100 \%)$ & $39(98 \%)$ & 0.31 \\
\hline & Total visible blood & $7(18 \%)$ & $2(5 \%)$ & 0.0001 \\
\hline & $\begin{array}{l}\text { Mean small fragment } \\
\text { (\% of total) }\end{array}$ & $\begin{array}{l}9.7(6.4) \\
60 \%\end{array}$ & $\begin{array}{l}10.9(7.1) \\
73 \%\end{array}$ & 0.43 \\
\hline & $\begin{array}{l}\text { Mean medium fragment } \\
\text { (\% of total) }\end{array}$ & $\begin{array}{l}2.7(1.9) \\
18 \%\end{array}$ & $\begin{array}{l}2.1(2.2) \\
13 \%\end{array}$ & 0.2 \\
\hline & $\begin{array}{l}\text { Mean long fragment } \\
\text { (\% of total) }\end{array}$ & $\begin{array}{l}3.0(2.0) \\
22 \%\end{array}$ & $\begin{array}{l}2.4(2.8) \\
14 \%\end{array}$ & 0.27 \\
\hline & Mean oength of the longest piece & $13.2(5.1)$ & $10.8(6.8)$ & 0.08 \\
\hline & Mean aggregate specimen length & $76.5(35.4)$ & $66.9(47.8)$ & 0.31 \\
\hline \multirow[t]{7}{*}{ Post-processing } & Mean portal tracts & $7.4(5.9)$ & $6.1(7.2)$ & 0.28 \\
\hline & \multicolumn{2}{|l|}{ Degree of fragmentation } & & \\
\hline & $\begin{array}{l}\text { Mean small fragment } \\
\text { (\% of total) }\end{array}$ & $\begin{array}{l}20.7(14.4) \\
82 \%\end{array}$ & $\begin{array}{l}29.9(17.9) \\
94 \%\end{array}$ & 0.01 \\
\hline & $\begin{array}{l}\text { Mean medium fragment } \\
\text { (\% of total) }\end{array}$ & $\begin{array}{l}2.1(1.9) \\
11 \%\end{array}$ & $\begin{array}{l}1.4(1.7) \\
5 \%\end{array}$ & 0.09 \\
\hline & $\begin{array}{l}\text { Mean long fragment } \\
\text { (\% of total) }\end{array}$ & $\begin{array}{l}1.0(1.3) \\
6 \%\end{array}$ & $\begin{array}{l}0.4(0.9) \\
1 \%\end{array}$ & 0.01 \\
\hline & Mean length of the longest piece & $9.1(4.5)$ & $6.6(4.7)$ & 0.02 \\
\hline & Mean aggregate specimen length & $61.0(30.4)$ & $48.1(32.2)$ & 0.07 \\
\hline \multirow{3}{*}{$\begin{array}{l}\text { Questionable tissue staging } \\
\text { parameters }\end{array}$} & Total with questionable portal inflammation & 0 & $5(13 \%)$ & 0.02 \\
\hline & Total with questionable portal fibrosis & $1(2.5 \%)$ & $6(15 \%)$ & 0.04 \\
\hline & Total with questionable centrilobular fibrosis & $1(2.5 \%)$ & $4(10 \%)$ & 0.17 \\
\hline \multicolumn{5}{|c|}{$\begin{array}{l}\text { G, gauge; SD, standard deviation; Cl, confidence interval } \\
1 \text { Chi Square testing was used for categorical variables } \\
2 \text { Mann Whitney U test was used for continuous variables } \\
{ }^{3} \text { Fisher's exact test was used for tissue staging parameters }\end{array}$} \\
\hline
\end{tabular}

\section{Outcomes}

For our primary outcome of tissue adequacy, 35/40 (88\%) of biopsies obtained by the 19G FNA needle were adequate when compared with $27 / 40$ (68\%) of $22 \mathrm{G}$ FNB samples $(P=0.03)$, which allowed for a clinical diagnosis to be obtained. Components of this primary outcome included $32 / 40$ (80\%) of $19 \mathrm{G}$ FNA samples contained $\geq 5$ portal structures, compared to $23 /$ $40(58 \%)$ of $22 \mathrm{G}$ FNB samples $(P=0.03)$. An additional component of this primary outcome included length of the longest piece $\geq 15 \mathrm{~mm}$, present in 15/40 (38\%) of 19G FNA samples versus $11 / 40(28 \%)$ of $22 \mathrm{G}$ FNB samples $(P=0.34)$. These outcomes were evaluated after tissue processing.

When evaluating our secondary outcomes, please refer to - Table 2 for a complete list of results. There was a significantly higher proportion of samples in the 19G FNA samples (7/40, $18 \%)$ that had visible blood clots in biopsy prior to processing as compared to the 22G FNB samples $(240,5 \%),(P<0.01)$. The mean number of portal structures obtained per needle, was 7.4 for the 19G FNA samples and 6.1 for the 22G FNB samples $(P=0.28)$, after processing.

Samples analyzed prior to processing demonstrated no difference in secondary outcomes ( $\triangleright$ Table 2 ). Specifically, the aggregate spciemen length was 76.5 and 66.9 for the $19 \mathrm{G}$ and $22 \mathrm{G}$, before processing $(P=0.31)$. Post-processing the aggregate specimen length was 61.0 and $48.1(P=0.07)$ for the 19G and $22 \mathrm{G}$ needles, respectively. After specimen processing, mean length of the longest piece was greater for the 19G FNA when compared with the $22 \mathrm{G}$ FNB samples $(9.1 \mathrm{~mm}$ versus $6.6 \mathrm{~mm}$, respectively; $P=0.02$ ) ( $\triangleright$ Fig. 2 ). 


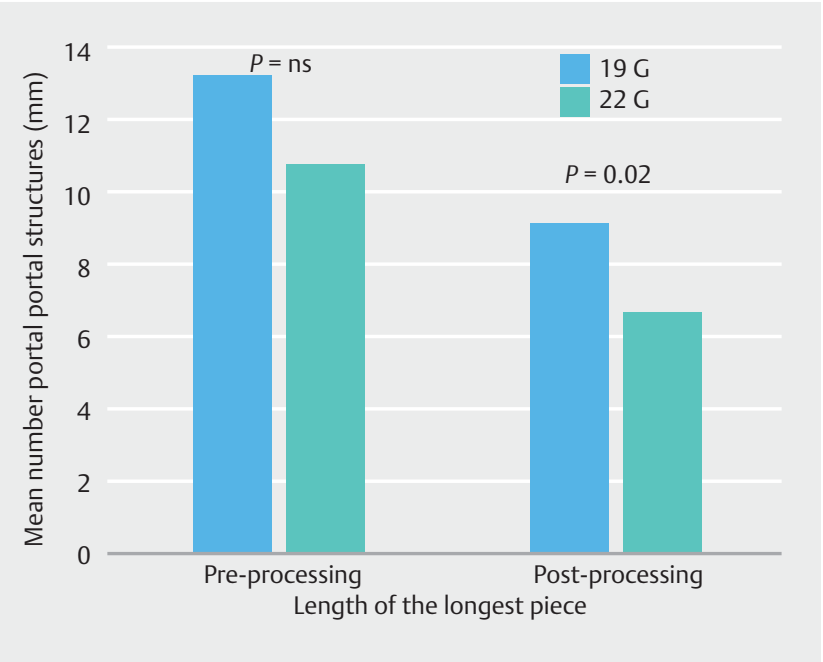

- Fig. 2 Comparison of portal structures, pre- and post-processing length of the longest piece for 19G FNA versus 22G FNB EUS-LB needles. Bars on left corresponded to 19G FNA needle and right bars 22G FNB. There was no significant difference in portal structures or pre-processing length of the longest piece, but there was a statistical difference $(P=0.02)$ for post-processing length of the longest piece. EUS-LB, endoscopic ultrasound-guided liver biopsy; g, gauge; ns, not significant.

The proportion of small, medium and large fragments both pre- and post-processing also was evaluated ( $\triangleright$ Fig. 3 and - Fig. 4). Please see $>$ Table 2 for a listing of pre-processing results. Post-processing evaluation demonstrated a larger mean total number of small fragments in the 22G FNB as compared with the 19G FNA samples (29.9 (90\%) versus 20.7 (80\%), respectively; $P=0.01$ ). There were more mean total post-processing large fragments for the 19G FNA samples versus the $22 \mathrm{G}$ FNB samples $(6 \%$ versus $1 \% P=0.01$ ). Otherwise there were no significant differences in post-processing medium fragments.

Pathologic scoring was analyzed on post-processing samples. Of the 19G FNA samples, 26/40 (65\%) had definite portal inflammation with zero questionable for inflammation, the remaining $14 / 40$ (35\%) did not have portal inflammation. This was compared with $24 / 40$ (60\%) of 22G FNB samples definite for portal inflammation, $5 / 40(13 \%)$ questionable $(P=0.02)$ and $16 / 40(60 \%)$ without inflammation. Portal fibrosis was present in 20/40 (50\%) and absent in 20/40 (50\%) of 19G FNA samples, with $1 / 40$ questionable (5\%). Contrarily, 22/40 (55\%) for 22G FNB sample had portal fibrosis, 18/20 (45\%) did not, 6/ $40(15 \%)$ which were questionable $(P=0.04)$. There were no diffences in centrilobular fibrosis determination between samples ( Table 2).

\section{Adverse events}

One patient who was randomized to the initial puncture with the $22 \mathrm{G}$ experienced pain after her procedure, but was discharged from the endoscopy suite without pain. Pain was present during the follow-up phone call 1 day after the procedure, and the patient was instructed to go to the emergency depart-

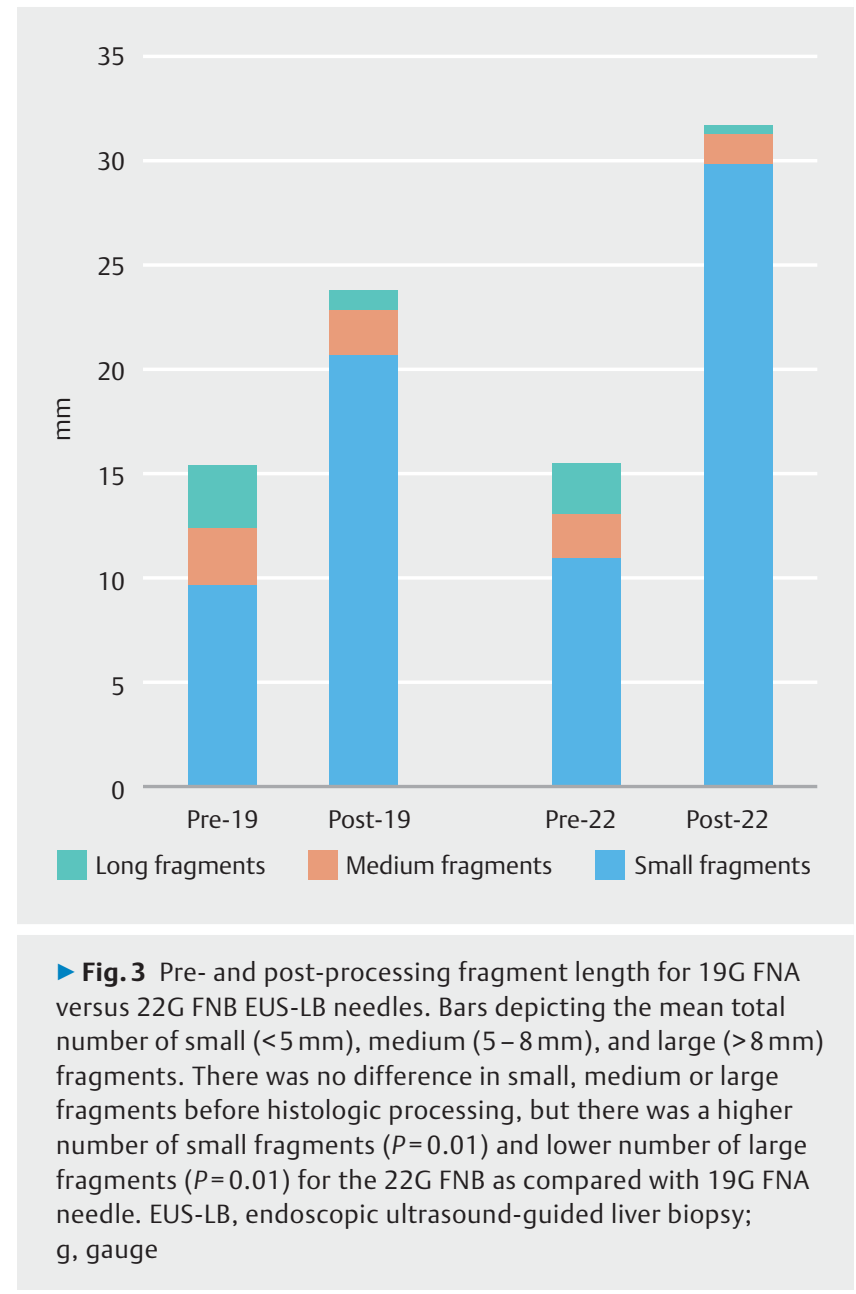

ment. The patient subsequently underwent laboratory testing, which revealed stable hemoglobin, and computed tomographic imaging showed no visible hemorrhage, perforation or abscess. This patient was discharged from the emergency department and remained pain-free during 7-day follow-up. There were no serious adverse events or deaths at 1-day and 7-day follow-up.

\section{Discussion}

Traditionally, liver biopsies have been performed using a $14 \mathrm{~g}$ or $16 \mathrm{~g}$ needle, utilizing percutaneous or surgical means $[6,7,20$ 30]. In recent years, use of EUS as a method for procuring liver tissue has gained traction with excellent tissue yields [8,9]. Using the EUS-LB method had various added benefits including concomitant examination of the upper gastrointestinal tract for additional pathology (i.e. for varices), lack of limitation in tissue procurment caused by increased body habitus, and direct avoidance of interveining vascular structures [8-17]. Our group had also compared tissue yields of percutaneous, tranjugular, and EUS-LB and found that EUS-LB tissue was comparable, if not slightly superior to these other methods [13].

As a 19G FNA needle is commonly used, to our knowledge, this is the first in vivo study to prospectively evaluate a smaller needle for obtaining EUS-guided parenchymal liver biopsies for 


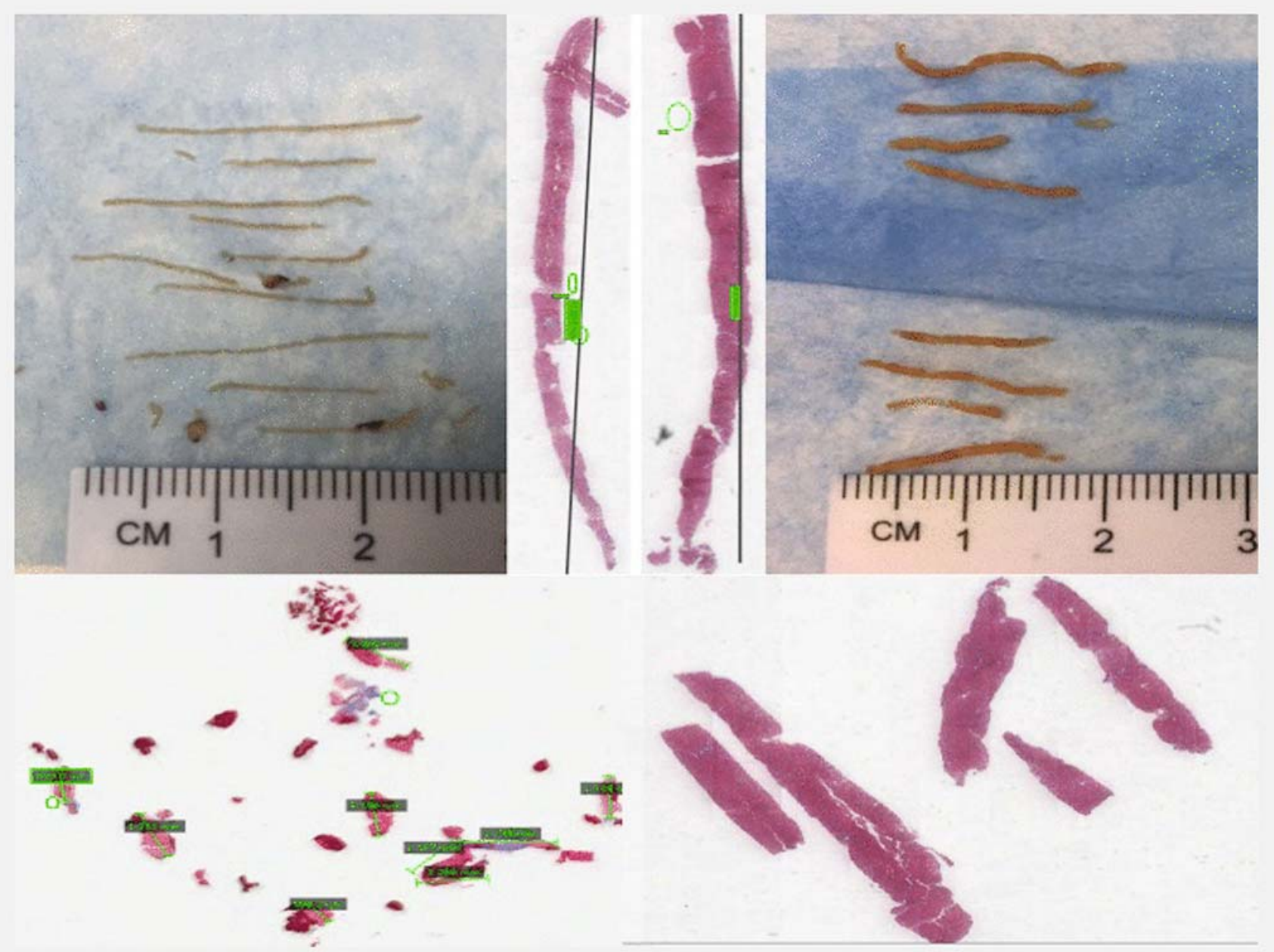

- Fig. 4 19G FNA and 22G FNB pre-processing gross images and post-processing histology.Upper panels (left to right) 22G FNB specimen gross image, post-processing histology image,19G FNA specimen post-processing histology image, 19G FNA specimen gross image. Lower panels (left to right) 22G FNB post-processing histology demonstrating fragmentation, 19G FNA post-processing histology with limited fragmentation.

clinical use $[8-19,31]$. By performing this study in a prospective and randomized fashion, and by using a blinded pathologic interpretation, we were able to evaluate the usefulness of a $22 \mathrm{G}$ FNB needle in live humans. The resulting data demonstrated that the 22G FNB needle can indeed provide adequate LB specimens, but in a smaller proportion of cases compared to the 19G FNA needle.

Interestingly, no differences in pre-processing tissue parameters were found between the 22G FNB needle and 19G FNA needle. It appears that after tisue processing, the 22G FNB samples suffered one total specimen loss (as evident by the equivalent aggregate specimen length), they are more delicate and prone to fragmentation during specimen processing (via lower post-processing length of the longest piece, higher small, and lower large fragments) as compared with the 19G FNA. That being said, the number of 19G FNA samples containing $\geq 5$ portal structures, a measure of tissue adequacy, were significantly higher than those from the 22G FNB samples. It is this finding that likely led to higher tissue adequacy for the 19G FNA versus 22G FNB samples.

A recent study compared various needle sizes and types for EUS-LB performed on a cadaveric liver [31]. In this ex vivo setting, the 19G FNA needle yielded the highest number of portal tracts when compared with a $22 \mathrm{G} \mathrm{FNB}$ and 2 different percutaneous $18 \mathrm{G}$ needles. Interestingly, the 22G FNB needle demonstrated an increased number of portal tracts when compared with the first $18 \mathrm{G}$ needle, but not a second $18 \mathrm{G}$ needle. This study also demonstrated that the degree of fragmentation (divided by variable core lengths $\geq 15 \mathrm{~mm}, 15-5 \mathrm{~mm}$, and $\leq 5 \mathrm{~mm}$ ) was equivalent for the 19G FNA and 22G FNB needles. However, the fragmentation measurements in the ex-vivo study were done only before histologic processing. Our study also showed that before histologic processing, there were similar metrics for the size of the liver cores between the 19G FNA and 22G FNB needles. Yet by measuring fragment lengths after histological processing, we demonstrated that the 22G FNB needle biopsies fragmented significantly more afterward. This 
likely contributed to the larger number of "inadequate" samples in the smaller needle samples.

Liver biopsy interpretation has necessarily changed over the last two decades [5-7,20-30]. In the past, very large-gauge needles were used, delivering longer and wider tissue cores. These cores were essentially the "gold standard" from which histological interpretation was based. In recent years, much of the liver biopsy workload has transitioned to the interventional radiology (IR) department, which had easier ability to perform image-guided biopsy [1,30]. Even for liver biopsies done in IR, there has been a shift away from the larger needles to smaller needles, which are $18 \mathrm{G}$ to $20 \mathrm{G}$. As a result, the pathologist had significantly smaller liver cores to interpret than before. Some histologic findings can be assessed from small fragments (e. g. steatosis) where others required more intact cores to interpret (e.g., steatohepatitis). Specimen evaluation was more difficult with fragmented specimens, for example, evaluation of portal inflammation. Our data demonstrated a higher degree of difficulty for the pathologists to make a definite decision regarding portal inflammation and portal fibrosis for the 22G FNB samples than 19G, but not centrilobular fibrosis. Therefore, it appeared that more specimen fragmentation of the 22G FNB samples led to higher diagnostic uncertainty for these pathologic parameters, as also contributing to lower tissue adequacy.

Strengths of this study included the prospective and randomized in vivo human design. Another strength was use of blinded expert pathology interpretation, which allowed for valid specimen interrogation while limiting bias. In addition, our pre- and post-processing analysis opens a window on the importance of addressing tissue fragmentation. Potential limitations of this study included that the endoscopist could not be blinded to needle type. Another important consideration was that this was a single-center experience conducted by endosonographers with extensive experience in EUS-LB, and a pathologist with comparable experience in interpreting EUS-LB slides. The tissue yields may not be generalizable to endosonogra-

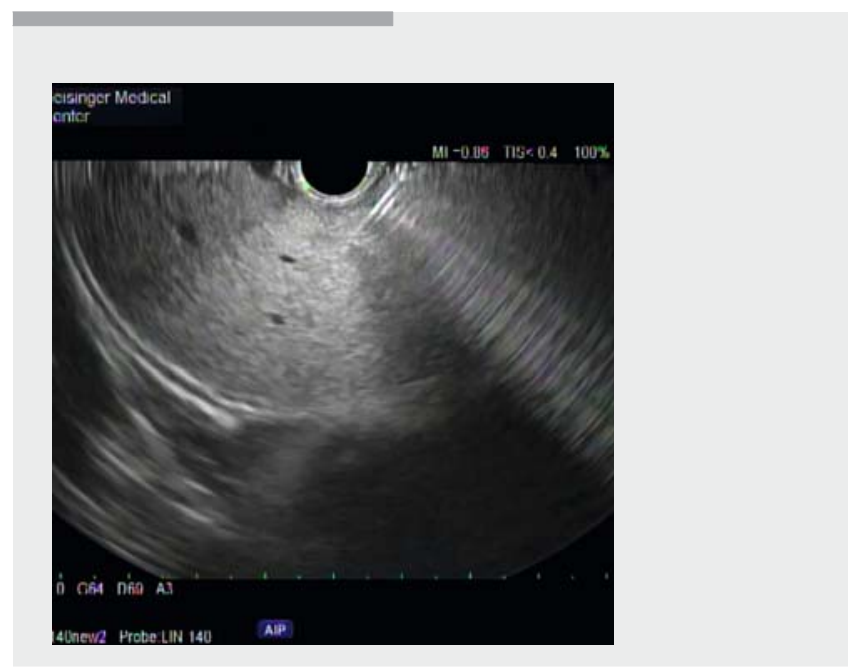

Video 1 Video demonstrating our EUS-guided liver biopsy technique. phers with less experience with EUS-LB, or their pathologists, who may not be used to interpreting these specimens. The study group also regonized the possibility of our cross-over design inducing a carrier effect. This theory may have impacted outcomes such as bloodiness, which could have been more prevalent for all second passes, but was not present when evaluating our data. It is important to note that the mean BMI of this cohort was 31.2, which is termed obese, which did not limit tissue procurement for EUS liver biopsy. This could theoretically have influenced study outcomes, however, it was uniform across our population. Finally, as we were comparing two separate needle types (FNA nd FNB) as well as gauges, differences stated in this study may be the results of both of these variables and not just needle gauge.

\section{Conclusion}

This randomized trial with a blinded outcome assessor demonstrated that tissue adequacy was higher for the 19G FNA versus 22G FNB core needle EUS-LB specimens, allowing for more cases delivering a clinical diagnosis. Although, aggregate specimen length and length of the longest piece were similar between the needles before tissue processing in the histology laboratory, higher post-processing tissue fragmentation was seen with the 22G FNB needle as well as higher uncertainty for evaluation of portal inflammation and fibrosis. Given this information, the authors recommend using a $19 \mathrm{G}$ needle for EUSguided liver biopsy.

\section{Acknowledgements}

The authors of this study would like to thank our endoscopy nurses and technicians for their assistance in this project. The authors of this study would like to thank Dustin Lin for assisting with data extraction.

\section{Competing interests}

Dr. Johal is a consultant for Boston Scientific Corporation. Dr. Khara is a consultant for Covidien-Metronic. Dr. Diehl is a consultant for Boston Scientific Corporation and Olympus.

References

[1] Rockey DC, Caldwell SH, Goodman ZD et al. American Association for the Study of Liver Diseases. Liver biopsy. Hepatology 2009; 49: 1017 1044

[2] Rockey DC, Bissell DM. Noninvasive measures of liver fibrosis. Hepatology 2006; 43: S113-20

[3] Rockey DC. Non-invasive assessment of liver fibrosis and portal hypertension with transient elastography. Gastroenterology 2008; 134 : $8-14$

[4] Ziol M, Handra-Luca A, Kettaneh A et al. Noninvasive assessment of liver fibrosis by measurement of stiffness in patients with chronic hepatitis C. Hepatology 2005; 41: 48 - 54

[5] Sherlock S, Dick R, Van Leeuwen DJ. Liver biopsy today. The Royal Free Hospital experience. J Hepatol 1985; 1: 75-85 
[6] Eisenberg E, Konopniki M, Veitsman E et al. Prevalence and characteristics of pain induced by percutaneous liver biopsy. Anesth Analg 2003; 96: $1392-1396$

[7] Perrault J, McGill DB, Ott B] et al. Liver biopsy: complications in 1000 inpatients and outpatients. Gastroenterology 1978; 74: 103-106

[8] Stavropoulos SN, Im GY, Jlayer Z et al. High yield of same-session EUSguided liver biopsy by 19-gauge FNA needle in patients undergoing EUS to exclude biliary obstruction. Gastrointest Endosc 2012; 75: $310-318$

[9] Diehl DL, Johal AS, Khara KS et al. Endoscopic ultrasound-guided liver biopsy: a multicenter experience. Endosc Int Open 2015; 3: E1 -E6

[10] Nakanishi Y, Mneimneh WS, Sey M et al. One hundred thirteen consecutive transgastric liver biopsies for hepatic parenchymal diseases: a single-institution study. Am J Surg Pathol 2015; 39: 968 - 976

[11] Dewitt J, McGreevy K, Cummings O et al. Initial experience with EUSguided Tru-cut biopsy of benign liver disease. Gastrointest Endosc 2009; 69: 535- 542

[12] Sey MS, Al-Haddad M, Imperiale TF et al. EUS-guided liver biopsy for parenchymal disease: a comparison of diagnostic yield between two core biopsy needles. Gastrointest Endosc 2016; 83: 347-352

[13] Pineda JJ, Diehl DL, Miao CL et al. EUS-guided liver biopsy provides diagnostic samples comparable with those via the percutaneous or transjugular route. Gastrointestinal Endosc 2016; 83: 360 - 365

[14] Gleeson FC, Clayton AC, Zhang L et al. Adequacy of endoscopic ultrasound core needle biopsy specimen of nonmalignant hepatic parenchymal disease. Clin Gastroenterol Hepatol 2008; 6: 1437-1440

[15] Gor N, Salem SB, Jakate S et al. Histological adequacy of EUS-guided liver biopsy when using a 19-gauge non-Tru-Cut FNA needle. Gastrointest Endosc 2014; 79: 170-172

[16] Bhatia V, Hijoka S, Hara K et al. Endoscopic ultrasound description of liver segmentation and anatomy. Dig Endosc 2014; 26: 482 - 490

[17] Sey MS, Al-Haddad M, Imperiale TF et al. EUS-guided liver biopsy for parenchymal disease: a comparison of diagnostic yield between two core biopsy needles. Gastrointest Endosc 2016; 83: 347-352

[18] Early DS, Acosta RD, Chandrasekhara V et al. Adverse events associated with EUS and EUS with FNA. Gastrointest Endosc 2013; 77960 : $839-843$
[19] Cotton PB, Eisen GM, Aabakken L et al. A lexicon for endoscopic adverse events: report of an ASGE workshop. Gastrointest Endosc 2010; 71: $446-454$

[20] Firpi R], Soldevila-Pico C, Abdelmalek MF et al. Short recovery time after percutaneous liver biopsy: should we change our current practices? Clin Gastroenterol Hepatol 2005; 3: 926 - 929

[21] Stone MA, Mayberry JF. An audit of ultrasound guided liver biopsies: a need for evidence-based practice. Hepatogastroenterology 1996; 43: $432-434$

[22] McGill DB, Rakela J, Zinsmeister AR et al. A 21-year experience with major hemorrhage after percutaneous liver biopsy. Gastroenterology 1990; 99: $1396-1400$

[23] Janes $\mathrm{CH}$, Lindor KD. Outcome of patients hospitalized for complications after outpatient liver biopsy. Ann Intern Med 1993; 118: 96 - 98

[24] Piccinino F, Sagnelli E, Pasquale G et al. Complications following percutaneous liver biopsy. A multicentre retrospective study on 68,276 biopsies. J Hepatol 1986; 2: 165-173

[25] Huang JF, Hsieh MY, Dai CY et al. The incidence and risks of liver biopsy in non-cirrhotic patients: An evaluation of 3806 biopsies. Gut 2007; 56: $736-737$

[26] Cadranel JF, Rufat P, Degos F. Practices of liver biopsy in France: results of a prospective nationwide survey. For the Group of Epidemiology of the French Association for the Study of the Liver (AFEF). Hepatology 2000; 32: 477- 481

[27] Myers RP, Fong A, Shaheen AA. Utilization rates, complications and costs of percutaneous liver biopsy: a population-based study including 4275 biopsies. Liver Int 2008; 28: 705-712

[28] Bravo AA, Sheth SG, Chopra S. Liver biopsy. N Engl J Med 2001; 344: $495-500$

[29] Lebrec D, Goldfarb G, Degott C et al. Transvenous liver biopsy: an experience based on 1000 hepatic tissue samplings with this procedure. Gastroenterology 1982; 83: 338 - 340

[30] Bull H], Gilmore IT, Bradley RD et al. Experience with transjugular liver biopsy. Gut 1983; 24: $1057-1060$

[31] Schulman AR, Thompson CC, Odze R et al. Optimizing EUS-guided liver biopsy sampling: comprehensive assessment of needle types and tissue acquisition techniques. Gastrointest Endosc 2017; 85: 419426 
Appendix 1 Pathology Assessment System

\begin{tabular}{|l|l|l|l|l|l|l|l|l|l|l|}
\hline $\begin{array}{l}\text { Random- } \\
\text { ized \# }\end{array}$ & $\begin{array}{l}\text { Adequate for } \\
\text { diagnosis } \\
\text { (Yes or No) }\end{array}$ & $\begin{array}{l}\text { Steatosis } \\
\text { (Y, N, Questionable) }\end{array}$ & $\begin{array}{l}\text { portal inflammation } \\
\text { (Y, N, Questionable) }\end{array}$ & $\begin{array}{l}\text { fibrosis, portal } \\
\text { (Y, N, Questionable) }\end{array}$ \\
\hline \\
\hline
\end{tabular}

